\title{
Suppressive effect of formononetin on platelet- derived growth factor-BB-stimulated proliferation and migration of vascular smooth muscle cells
}

\author{
ZHUO CHEN, SUIXIN LIU, YING CAI, KANGLING XIE, WENLIANG ZHANG, \\ LEI DONG, YUAN LIU, FAN ZHENG, YAOSHAN DUN and NING LI \\ Department of Rehabilitation, Cardiac Rehabilitation Center, Xiangya Hospital of Central South University, \\ Changsha, Hunan 410008, P.R. China
}

Received January 26, 2015; Accepted February 26, 2016

DOI: $10.3892 / e t m .2016 .3514$

\begin{abstract}
Abnormal proliferation and migration of vascular smooth muscle cells (VSMCs) has been implicated in intimal hyperplasia, atherosclerosis and restenosis following percutaneous coronary intervention. Formononetin, a phytoestrogen extracted from the root of Astragalus membranaceus, has been widely used in Chinese tradition medicine due to its protective effects against certain symptoms of cancer, hypertension, inflammation, hypoxia-induced cytotoxicity and ovariectomy-induced bone loss. However, the effect of formononetin on platelet-derived growth factor (PDGF)-BB-induced proliferation and migration of VSMCs, as well as the underlying molecular mechanism, remains largely unclear. In the present study, treatment with formononetin significantly inhibited PDGF-BB-induced proliferation and migration of human VSMCs. Investigation into the underlying molecular mechanism revealed that the administration of formononetin suppressed PDGF-BB-stimulated switch of VSMCs to a proliferative phenotype. Furthermore, treatment with formononetin inhibited the PDGF-BB-induced upregulation of cell cycle-related proteins, matrix metalloproteinase (MMP2) and MMP9. In addition, the that administration of formononetin inhibited the phosphorylation of AKT induced by PDGF-BB in VSMCs. The present results suggest that formononetin has a suppressive effect on PDGF-BB-stimulated VSMCs proliferation and migration, which may occur partly via the inhibition of AKT signaling pathway. Therefore, formononetin may be useful for the treatment of intimal hyperplasia, atherosclerosis and restenosis.
\end{abstract}

Correspondence to: Professor Suixin Liu, Department of Rehabilitation, Cardiac Rehabilitation Center, Xiangya Hospital of Central South University, 87 Xiangya Road, Changsha, Hunan 410008, P.R. China

E-mail: csuliusuixin@163.com

Key words: formononetin, vascular smooth muscle cell, platelet-derived growth factor, proliferation, migration

\section{Introduction}

Under standard physiological conditions, vascular smooth muscle cells (VSMCs) generally remain in a quiescent state. However, in response to vascular damage or inflammatory stimulations, VSMCs may undergo phenotypic changes to an uncontrolled proliferating and migratory state (1). It has been well established that the abnormal proliferation and migration of VSMCs in arterial walls is crucial in the development and progression of cardiovascular disorders, including intimal hyperplasia, arteriosclerosis and restenosis following percutaneous coronary intervention (PCI) $(2,3)$.

Formononetin is an O-methylated isoflavone phytoestrogen extracted from the root of Astragalus membranaceus, which has been widely used in Chinese medicine for $>2,000$ years. A. membranaceus has various bioactivities, such as anti-viral, anti-oxidant, anti-tumor, anti-diabetes, anti-inflammation, anti-atherosclerosis, immunomodulation, hepatoprotection, hematopoiesis and neuroprotection (4-7). As an important component of A. membranaceus, formononetin has also been demonstrated to have various pharmacological effects. For instance, previous studies have indicated that formononetin is able to inhibit tumor cell proliferation, migration and invasion $(8,9)$, induce tumor cell apoptosis (10), attenuate hydrogen peroxide-induced retinal ganglion cell apoptosis (11), as well as mediate neuroprotection against cerebral ischemia/reperfusion (12).

Recent studies have demonstrated that formononetin may exert protective effects against cardiovascular disorders. Huh et al reported that formononetin promoted endothelial repair and wound healing (13). Zhu et al found that formononetin had neuroprotective effects against cerebral ischemia and reperfusion injury in rats, and improved cerebrovascular angiogenesis in human umbilical vein endothelial cells (14). However, to the best of our knowledge, the effect of formononetin on VSMCs has not previously been studied.

The aim of the present study was to investigate the effect of formononetin on PDGF-BB-stimulated VSMC proliferation and migration, in addition to elucidating the underlying mechanisms. 


\section{Materials and methods}

Materials and agents. Formononetin was purchased from TAOTU Biotech (Shanghai, China). Dulbecco's modified Eagle's medium (DMEM)/F12, fetal bovine serum (FBS), and BCA Protein Assay Kit were purchased from Life Technologies (Carlsbad, CA, USA). An enhanced chemiluminescence kit was purchased from Pierce Biotechnology (Thermo Fisher Scientific, Inc., Rockford, IL, USA). Recombinant human PDGF-BB, dimethyl sulfoxide (DMSO), MTT, bovine serum albumin (BSA) and radioimmunoprecipitation assay (RIPA) buffer were purchased from Sigma-Aldrich (St. Louis, MO, USA). A 24-well chamber was purchased from Corning, Inc., (Corning, NY, USA). Mouse monoclonal antibodies against smoothelin (1:100; ab8969), $\alpha$-smooth muscle actin ( $\alpha$-SMA; 1:200; ab7817), desmin (1:50; ab8470), cyclin D1 (1:50; ab6152), cyclin-dependent kinase 4 (CDK4; 1:200; ab75511), matrix metalloproteinase 2 (MMP2; 1:100; ab86607), MMP9 (1:100; ab58803), phospho-AKT, mouse AKT (1:100; ab105731) and glyceraldehyde 3-phosphate dehydrogenase (GAPDH; 1:50; ab8245), in addition to goat anti-mouse secondary antibody were obtained from Abcam (1:20,000; ab6785; Cambridge, MA, USA).

Cell culture. Human dermis VSMCs were purchased from ScienCell Research Laboratories (Carlsbad, CA, USA). VSMCs were cultured in DMEM/F12 medium with $10 \%$ FBS at $37^{\circ} \mathrm{C}$ in a humidified atmosphere of $95 \%$ air and $5 \% \mathrm{CO}_{2}$.

MTT assay. An MTT assay was performed for examining the cell proliferation. Three groups were established, as follows: Control group, VSMCs were without any treatment; PDGF-BB group, VSMCs were treated with PDGF-BB $(30 \mathrm{ng} / \mathrm{ml})$; and PDGF-BB + formononetin group, VSMCs were treated with PDGF-BB $(30 \mathrm{ng} / \mathrm{ml})$ and formononetin $(1 \mu \mathrm{M})$. Cells $\left(5 \times 10^{3}\right)$ in each group were seeded into 96 -well plates, and cultured for $0,12,24$ and $48 \mathrm{~h}$, respectively. Then, $10 \mu \mathrm{l}$ MTT $(10 \mathrm{mg} / \mathrm{ml})$ was added to the cells and incubated for $4 \mathrm{~h}$ prior to termination of the reaction by removing the supernatant and adding $100 \mu \mathrm{l}$ DMSO to dissolve the formazan product. Following incubation for $30 \mathrm{~min}$, the optical density of each well was measured at $570 \mathrm{~nm}$ using a plate reader (ELx808; BioTek Instruments, Inc., Winooski, VT, USA).

Scratch assay. Cell migration in each group was examined using a costar 24-well chamber (Corning Inc., Shanghai, China). The cells were counted under a CX23 microscope (Olympus, Tokyo, Japan). In brief, cell suspension $\left(5 \times 10^{5}\right.$ cells $\left./ \mathrm{ml}\right)$ was prepared in DMEM/F12 medium. In accordance with the manufacturer's instructions, $500 \mu \mathrm{l}$ DMEM/F12 with $10 \%$ FBS was added to the lower chamber, and $300 \mu 1$ cell suspension was added into the upper chamber. In the PDGF-BB group, PDGF-BB $(30 \mathrm{ng} / \mathrm{ml})$ was also added to the lower wells. In the PDGF-BB + formononetin group, the lower wells contained PDGF-BB $(30 \mathrm{ng} / \mathrm{ml})$ and formononetin $(1 \mu \mathrm{M})$. The relative migration in the PDGF-BB group is presented as the cell number ratio of PDGF-BB versus the control. Similarly, the relative migration in the PDGF-BB+formononetin group is presented as the cell number ratio of PDGF-BB+formononetin versus the control. After $24 \mathrm{~h}$ incubation at $37^{\circ} \mathrm{C}$ with $5 \%$
$\mathrm{CO}_{2}$, cells that had not migrated through the membrane were removed, while cells that had were stained with crystal violet dye (Beyotime Institute of Biotechnology, Haimen, China) for $30 \mathrm{~min}$, then rinsed with water and dried in air. The stained cells were counted and the relative cell migration was determined.

Western blot analysis. Cells were washed with phosphate-buffered saline once and $500 \mu 1$ RIPA buffer was added to lyse the cells. Cells were then centrifuged at $8,000 \mathrm{x} g$ for $10 \mathrm{~min}$ at $4^{\circ} \mathrm{C}$, and the supernatant containing the protein was collected. The concentration of protein was determined using a BCA Protein Assay kit, in accordance with the manufacture's instructions. Next, $50 \mu \mathrm{g}$ protein was run on a $12 \%$ SDS-PAGE gel (Beyotime Institute of Biotechnology) and blotted onto polyvinylidene difluoride membranes (Thermo Fisher Scientific, Inc.), which were blocked in 5\% BSA for $1.5 \mathrm{~h}$ at room temperature, followed by incubation overnight at $4^{\circ} \mathrm{C}$ with the indicated antibodies. The membranes were rinsed and incubated for $1 \mathrm{~h}$ at room temperature with the appropriate peroxidase-conjugated secondary antibodies. Chemiluminescent detection was performed using the enhanced chemiluminescence kit. The relative protein expression was analyzed by Image Pro Plus software version 6.0 (Media Cybernetics, Inc., Rockville, MD, USA) and presented as the density ratio of FSCN1 versus GAPDH.

Statistical analysis. Data is presented as the mean \pm standard deviation of at least three independent experiments. SPSS software, version 17.0 (SPSS, Inc., Chicago, IL, USA) was used for statistical analysis. One-way analysis of variance was used to analyze the differences between groups. $\mathrm{P}<0.05$ was considered to indicate as statistically significant difference.

\section{Results}

Formononetin inhibited PDGF-BB-stimulated proliferation and migration of VSMCs. The effects of formononetin on PDGF-BB-induced VSMCs proliferation and migration were investigated first. MTT assay data showed that the PDGF-BB treatment enhanced the proliferation of VSMCs compared with the control group, which was notably attenuated by the treatment with formononetin (Fig. 1A). These data suggest that formononetin inhibits PDGF-BB-stimulated VSMCs proliferation. Subsequently, the effect of formononetin on the PDGF-BB-stimulated migration of VSMCs was investigated. The results of a scratch assay showed that treatment with PDGF-BB significantly promoted VSMCs migration compared with the control group; however, formononetin significantly attenuated the upregulation of PDGF-BB-induced VSMC migration (Fig. 1B), suggesting that formononetin has an inhibitory effect on PDGF-BB-induced VSMCs migration.

Formononetin inhibited the PDGF-BB-induced phenotype change of VSMCs. Under normal physiological conditions, vascular smooth muscle cells (VSMCs) generally remain in a quiescent state. However, in response to vascular damage or inflammatory stimulation, VSMCs may undergo phenotypic changes to an uncontrolled proliferating and migratory state (1). Smoothelin, $\alpha$-SMA and desmin are markers for the 
quiescent phenotype of VSMCs (15). Therefore, the expression levels of these proteins were detected in each group. As shown in Fig. 2, administration of PDGF-BB significantly inhibited the protein expression levels of smoothelin, $\alpha$-SMA and desmin in VSMCs, which cause VSMCs to dedifferentiate into a proliferative phenotype. However, treatment with formononetin significantly attenuated the PDGF-BB-induced downregulation of $\alpha$-SMA, smoothelin and desmin protein expression in VSMCs (Fig. 2), suggesting that formononetin has an inhibitory effect on PDGF-BB-induced phenotype switch in VSMCs.

Formononetin inhibited the PDGF-BB-induced expression of cell cycle-related proteins in VSMCs. Cell cycle-related proteins, including CDK2, CDK4, cyclin D1 and cyclin E, are crucially involved in the regulation of cell cycle progression, as well as cell proliferation (16). It has been reported that formononetin has effects on the expression of cell cycle-related proteins (8). Therefore, the effect of formononetin on VSMCs proliferation may be associated with the expression levels of cell cycle-related proteins. Western blot analysis was conducted to determine the protein levels of CDK2, CDK4, cyclin D1, and cyclin E in each group. As shown in Fig. 3, administration of PDGF-BB significantly enhanced the protein expression levels of CDK2, CDK4, cyclin D1, and cyclin E in VSMCs; however, treatment with formononetin significantly suppressed PDGF-BB-stimulated upregulation of CDK2, CDK4, cyclin D1 and cyclin E, suggesting that the suppressive effect of formononetin on PDGF-BB-induced VSMCs proliferation may partly occur via the inhibition of the expression of cell cycle-related proteins.

Formononetin suppressed PDGF-BB-induced upregulation of MMP2 and MMP9 in VSMCs. It has been well established that MMP2 and MMP9 play key roles in the regulation of cell migration (9). Therefore, the protein expression levels of MMP2 and MMP9 were determined in the VSMCs in each group. As shown in Fig. 4, MMP2 and MMP9 were significantly upregulated following treatment with PDGF-BB, which was significantly attenuated by treatment with formononetin. These results suggest that the suppressive effect of formononetin on PDGF-BB-induced VSMCs migration is mediated by the inhibition of MMP2 and MMP9 protein expression.

Formononetin suppressed PDGF-BB-induced activation of AKT signaling in VSMCs. AKT signaling pathway has been implicated in the regulation of cell proliferation and migration, in addition the expression of cell cycle-related proteins and MMPs $(8,17)$. Accordingly, the activity of AKT signaling in VSMCs was evaluated in the present study. As shown in Fig. 5, the phospho-AKT protein expression was significantly upregulated by treatment with PDGF-BB, when compared with the control group, suggesting that PDGF-BB is able to activate the AKT signaling pathway. However, treatment with formononetin effectively suppressed PDGF-BB-stimulated upregulation of phospho-AKT protein level in VSMCs, suggesting that formononetin is able to inhibit PDGF-BB-induced activation of AKT signaling in VSMCs.

\section{Discussion}

The results of the present study suggest that formononetin exerted an inhibitory effect against PDGF-BB-stimulated VSMCs proliferation and migration. Formononetin was able to inhibit the PDGF-BB-stimulated change of VSMCs into a proliferative phenotype, suppressed the enhanced expression of cell cycle-related proteins and MMPs, in addition to downregulating the activity of AKT signaling.

VSMCs are continually stimulated by the biochemical components in the blood compartment, which may affect their phenotypes such as cell proliferation and migration. Thus VSMCs are involved in the physiological and pathological processes in the vascular wall $(18,19)$. For example, following vascular injury various cytokines, including PDGF-BB, are released by endothelial cells and macrophages. These stimulate the abnormal proliferation and migration VSMCs, a key promoter in the initiation of intimal hyperplasia, which can further lead to arteriosclerosis and restenosis following PCI (20-22). Therefore, inhibition of PDGF-BB-induced VSMCs proliferation and migration is crucial for the prevention of atherosclerosis and restenosis. Formononetin has been shown to inhibit the proliferation of multiple types of cancer cells. Li et al showed that formononetin inhibited the proliferation of human prostate cancer cells via inducing cell cycle arrest (8). Liu et al showed that formononetin suppressed proliferation while inducing the apoptosis of osteosarcoma cells (23). However, the effects of formononetin on VSMCs proliferation have been hitherto unclear. Herein, it was reported that formononetin suppressed PDGF-BB-stimulated VSMCs proliferation. Furthermore, the results indicate that PDGF-BB could induce VSMCs to dedifferentiate into a proliferative phenotype, as suggested by the downregulation of SMA, smoothelin and desmin, which is consistent with previous studies $(16,24)$. However, treatment with formononetin attenuated the PDGF-BB-induced downregulation of SMA, smoothelin and desmin, indicating that formononetin inhibited the PDGF-BB-induced phenotype change in VSMCs.

Cell cycle-related proteins such as cyclin D1, cyclin E, CDK2 and CDK4 are crucially involved in the regulation of cell proliferation. Previous studies have shown that these cell cycle-related proteins are associated with PDGF-BB-stimulated VSMCs proliferation $(25,26)$. Furthermore, it has been reported that formononetin is able to mediate the expression of these proteins. For example, formononetin promotes cell cycle arrest via the downregulation of cyclin D1 and CDK4 expression in human prostate cancer cells (8). The present results suggest that treatment with formononetin significantly attenuated the PDGF-BB-stimulated upregulation of cyclin D1, cyclin E, CDK2 and CDK4.

MMP2 and MMP9 have been shown to play key roles in the regulation of VSMCs migration. For instance, Ding et al observed that resistin stimulated MMP-2 and MMP-9 expression and VSMC migration, while neutralizing antibodies against MMP-2 and MMP-9 effectively reversed resistin-stimulated VSMC migration (27). In addition, MMP2 and MMP9 are involved in intimal hyperplasia. Guo et al found that neointimal hyperplasia was reduced in $\mathrm{MMP}^{-/-}$or $\mathrm{MMP}^{-/-}$mice after femoral artery injury (28). In the present study, it was shown that treatment of PDGF-BB increased the expression levels 
A

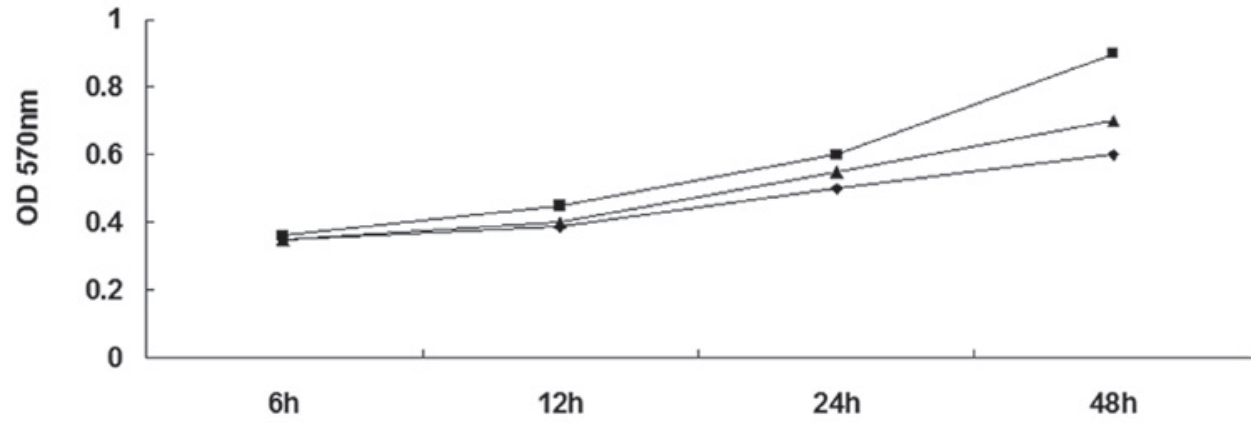

B

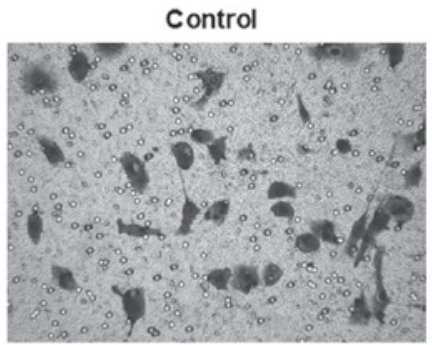

PDGF-BB

PDGF-BB+formononetin
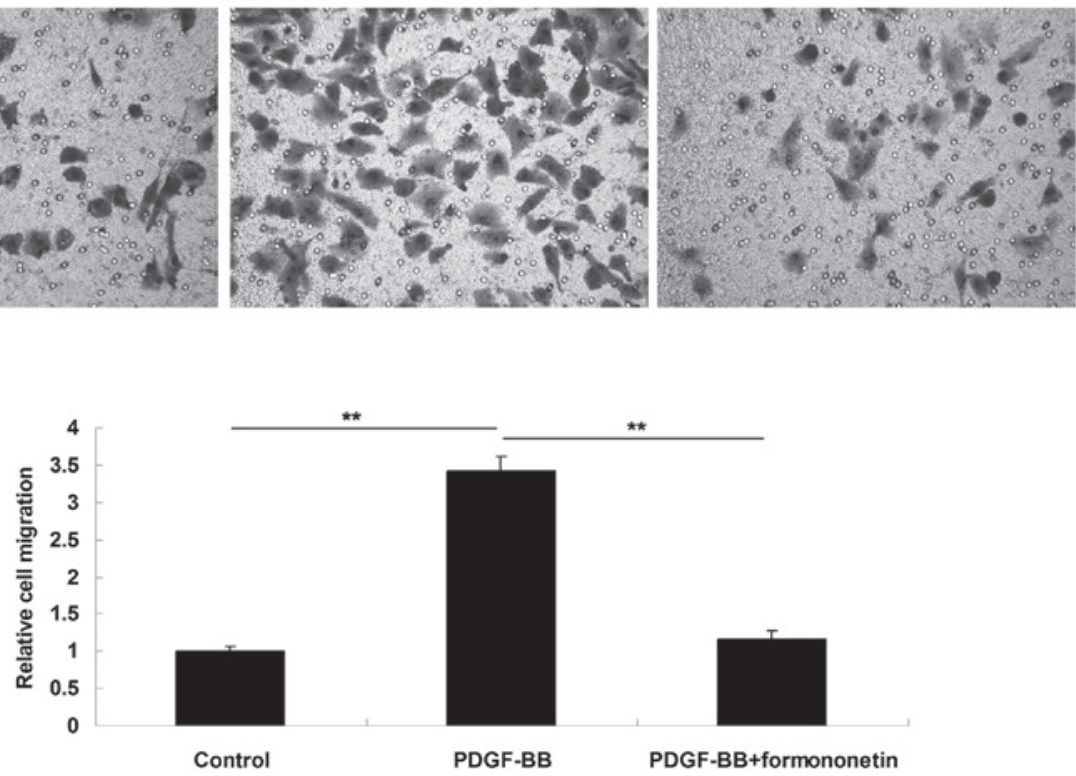

Figure 1. Formononetin inhibited PDGF-BB-induced proliferation and migration of vascular smooth muscle cells (VSMCs). (A) MTT assay was performed to determine VSMC proliferation. (B) Transwell assay was performed to determine VSMC migration. ${ }^{* * *} \mathrm{P}<0.01$. One way analysis of variance was used to analyze the differences between groups. OD, optical density; PDGF-BB, platelet-derived growth factor-BB.

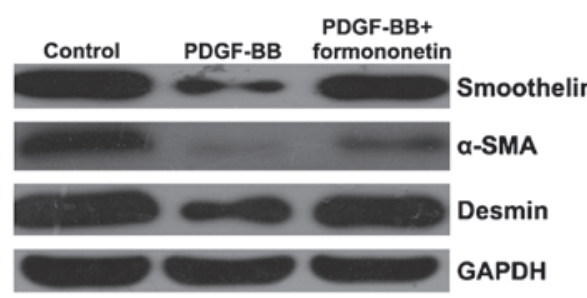

- Control $\square$ PDGF-BB $\square$ PDGF-BB+formononetin

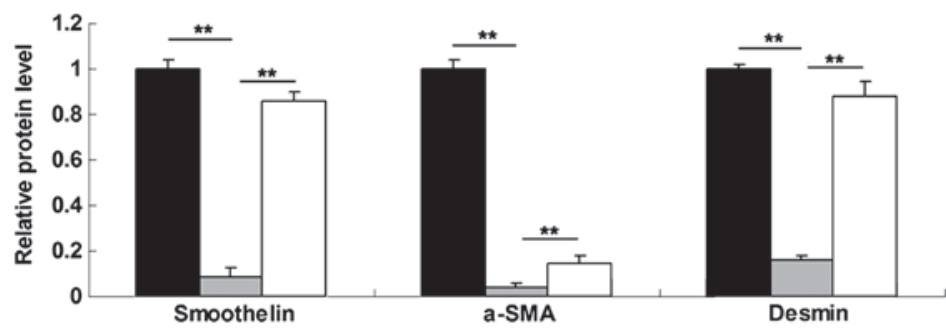

Figure 2. Formononetin inhibited PDGF-BB-induced phenotype switch of vascular smooth muscle cells. Western blot analysis was conducted to evaluate the protein expression of smoothelin, $\alpha$-SMA and desmin. GAPDH was used as an internal reference. ${ }^{* *} \mathrm{P}<0.01$. One way analysis of variance was used to analyze the differences between groups. PDGF-BB, platelet-derived growth factor-BB; $\alpha$-SMA, $\alpha$-smooth muscle actin. 


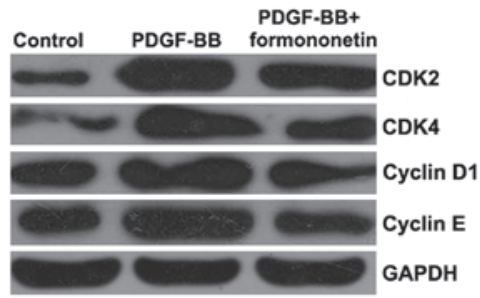

- Control $\square$ PDGF-BB $\square$ PDGF-BB+formononetin

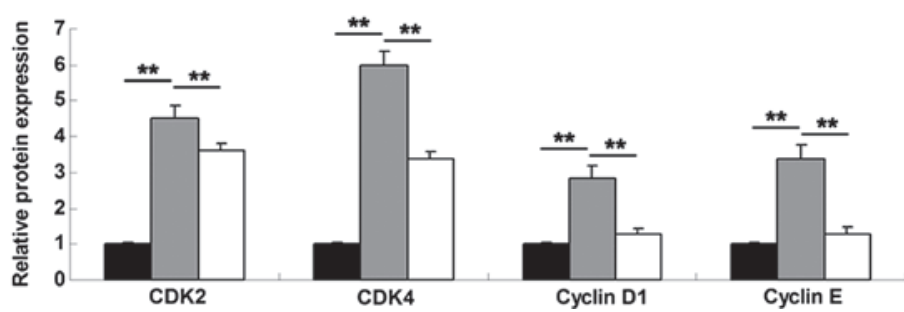

Figure 3. Formononetin suppressed PDGF-BB-induced upregulation of cell cycle-related proteins in vascular smooth muscle cells. Western blot analysis was performed to determine the expression levels of $\mathrm{CDK} 2, \mathrm{CDK} 4$, cyclin $\mathrm{D} 1$ and cyclin E. GAPDH was used as an internal reference. ${ }^{* * *} \mathrm{P}<0.01$. One way analysis of variance was used to analyze the differences between groups. PDGF-BB, platelet-derived growth factor-BB; CDK, cyclin-dependent kinase.

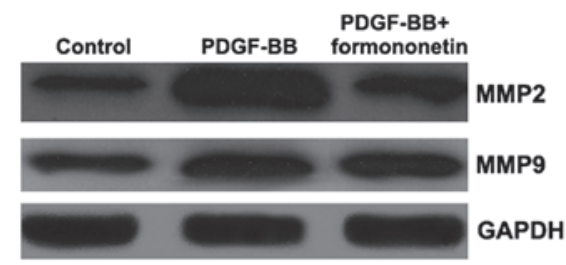

a Control $\square$ PDGF-BB $\square$ PDGF-BB+formononetin

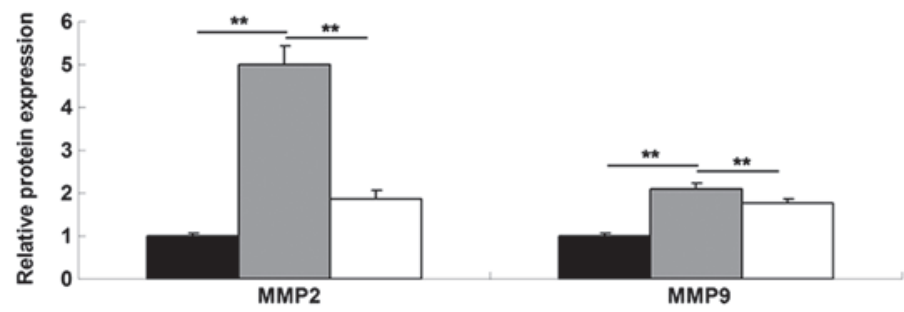

Figure 4. Formononetin inhibited PDGF-BB-induced upregulation of MMP2 and MMP9 in vascular smooth muscle cells. Western blot analysis was performed to determine the protein expression levels of MMP2 and MMP9. GAPDH was used as an internal reference. ${ }^{* *} \mathrm{P}<0.01$. One way analysis of variance was used to analyze the differences between groups. PDGF-BB, platelet-derived growth factor-BB; MMP, matrix metalloproteinase.
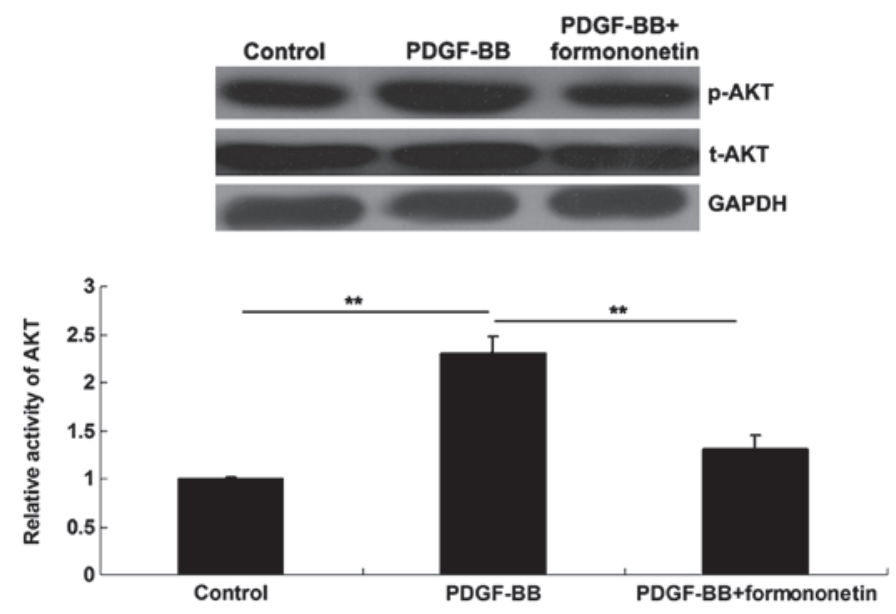

Figure 5. Formononetin suppressed PDGF-BB-induced activation of AKT signaling in VSMCs. Western blot analysis was performed to determine the protein expression of $\mathrm{p}$-AKT and $\mathrm{t}-\mathrm{AKT}$ in each group. GAPDH was used as an internal reference. ${ }^{* *} \mathrm{P}<0.01$. One way analysis of variance was used to analyze the differences between groups. PDGF-BB, platelet-derived growth factor-BB; p-AKT, phosphorylated AKT; t-AKT, total-AKT. 
of MMP2 and MMP9, which is consistent with the findings of Guo et al (28). Moreover, treatment with formononetin markedly suppressed the PDGF-BB-stimulated upregulation of MMP2 and MMP9, suggesting that the suppressive effect of formononetin on PDGF-BB-stimulated VSMCs migration is partly mediated via the inhibition of MMP2 and MMP9 expression.

AKT signaling has been implicated in various cellular biological processes, such as cell survival, apoptosis, cell cycle progression and angiogenesis, in addition to cell migration and invasion (29,30). In addition, it has been demonstrated that AKT signaling is involved in regulating the expression of a number of cell cycle-related proteins and MMPs $(9,31,32)$. Therefore, the activity of AKT signaling after treatment with PDGF-BB with or without formononetin was evaluated in the present study. Treatment with PDGF-BB appeared to enhance the phosphorylated protein level of AKT, indicating that the activity of AKT signaling was upregulated, which is consistent with previous studies $(15,33,34)$. However, treatment with formononetin effectively suppressed PDGF-BB-stimulated upregulation of phospho-AKT protein level in VSMCs, suggesting that formononetin is able to inhibit PDGF-BB-induced activation of AKT signaling in VSMCs.

In conclusion, the results of the present study demonstrate that treatment with formononetin is able to inhibit PDGF-BB-induced VSMC proliferation and migration via the inhibition of phenotype switch, expression of cell cycle-related proteins and MMPs, in addition to the activity of AKT signaling. Therefore, formononetin may require further investigation as a potential treatment for intimal hyperplasia, atherosclerosis and restenosis following PCI.

\section{Acknowledgements}

This study was supported by the Fundamental Research Funds for the Central University of Central South University (grant no. 2013zzts088).

\section{References}

1. Rodríguez AI, Csányi G, Ranayhossaini DJ, Feck DM, Blose KJ, Assatourian L, Vorp DA and Pagano PJ: MEF2B-Nox1 signaling is critical for stretch-induced phenotypic modulation of vascular smooth muscle cells. Arterioscler Thromb Vasc Biol 35: 430-438, 2015.

2. Chiong M, Cartes-Saavedra B, Norambuena-Soto I, Mondaca-Ruff D, Morales PE, García-Miguel M and Mellado R: Mitochondrial metabolism and the control of vascular smooth muscle cell proliferation. Front Cell Dev Biol 2: 72, 2014.

3. Choe N, Kwon JS, Kim JR, Eom GH, Kim Y, Nam KI, Ahn Y, Kee HJ and Kook H: The microRNA miR-132 targets Lrrfipl to block vascular smooth muscle cell proliferation and neointimal hyperplasia. Atherosclerosis 229: 348-355, 2013.

4. Jin M, Zhao K, Huang Q and Shang P: Structural features and biological activities of the polysaccharides from Astragalus membranaceus. Int J Biol Macromol 64: 257-266, 2014.

5. Agyemang K, Han L, Liu E, Zhang Y, Wang T and Gao X: Recent Advances in Astragalus membranaceus anti-diabetic research: Pharmacological effects of its phytochemical constituents. Evid Based Complement Alternat Med 2013: 654643, 2013.

6. Zhang J, Xie X, Li C and Fu P: Systematic review of the renal protective effect of Astragalus membranaceus (root) on diabetic nephropathy in animal models. J Ethnopharmacol 126: 189-196, 2009.
7. Wu $F$ and Chen $X$ : A review of pharmacological study on Astragalus membranaceus (Fisch.) Bge. Zhong Yao Cai 27: 232-234, 2004 (In Chinese).

8. Li T, Zhao X, Mo Z, Huang W, Yan H, Ling Z and Ye Y: Formononetin promotes cell cycle arrest via downregulation of Akt/Cyclin D1/CDK4 in human prostate cancer cells. Cell Physiol Biochem 34: 1351-1358, 2014.

9. Zhou R, Xu L, Ye M, Liao M, Du H and Chen H: Formononetin inhibits migration and invasion of MDA-MB-231 and 4T1 breast cancer cells by suppressing MMP-2 and MMP-9 through PI3K/AKT signaling pathways. Horm Metab Res 46: 753-760, 2014.

10. Zhang X, Bi L, Ye Y and Chen J: Formononetin induces apoptosis in PC-3 prostate cancer cells through enhancing the Bax/Bcl-2 ratios and regulating the p38/Akt pathway. Nutr Cancer 66: 656-661, 2014.

11. Jia WC, Liu G, Zhang CD and Zhang SP: Formononetin attenuates hydrogen peroxide (H2O2)-induced apoptosis and NF- $\kappa \mathrm{B}$ activation in RGC-5 cells. Eur Rev Med Pharmacol Sci 18: 2191-2197, 2014.

12. Liang K, Ye Y, Wang Y, Zhang J and Li C: Formononetin mediates neuroprotection against cerebral ischemia/reperfusion in rats via downregulation of the $\mathrm{Bax} / \mathrm{Bcl}-2$ ratio and upregulation PI3K/Akt signaling pathway. J Neurol Sci 344: 100-104, 2014.

13. Huh JE, Nam DW, Baek YH, Kang JW, Park DS, Choi DY and Lee JD: Formononetin accelerates wound repair by the regulation of early growth response factor-1 transcription factor through the phosphorylation of the ERK and p38 MAPK pathways. Int Immunopharmacol 11: 46-54, 2011.

14. Zhu H, Zou L, Tian J, Lin F, He J and Hou J: Protective effects of sulphonated formononetin in a rat model of cerebral ischemia and reperfusion injury. Planta Med 80: 262-268, 2014.

15. Lee MH, Kwon BJ, Seo HJ, Yoo KE, Kim MS, Koo MA and Park JC: Resveratrol inhibits phenotype modulation by platelet derived growth factor-bb in rat aortic smooth muscle cells. Oxid Med Cell Longev 2014: 572430, 2014.

16. Chen Z, Cai Y, Zhang W, Liu X and Liu S: Astragaloside IV inhibits platelet-derived growth factor-BB-stimulated proliferation and migration of vascular smooth muscle cells via the inhibition of p38 MAPK signaling. Exp Ther Med 8: 1253-1258, 2014.

17. Guan BZ, Yan RL, Huang JW, Li FL, Zhong YX, Chen Y, Liu FN, Hu B, Huang SB and Yin LH: Activation of G Protein coupled estrogen receptor (GPER) promotes the migration of renal cell carcinoma via the PI3K/AKT/MMP-9 signals. Cell Adh Migr 0, 2015.

18. Salabei JK and Hill BG: Autophagic regulation of smooth muscle cell biology. Redox Biol 4: 97-103, 2015.

19. Qiu J, Zheng Y, Hu J, Liao D, Gregersen H, Deng X, Fan Y and Wang G: Biomechanical regulation of vascular smooth muscle cell functions: From in vitro to in vivo understanding. J R Soc Interface 11: 20130852, 2013.

20. Li H, Luo K and Hou J: Inhibitory effect of Puerariae radix flavones on platelet-derived growth factor-BB-induced proliferation of vascular smooth muscle cells via PI3K and ERK pathways. Exp Ther Med 9: 257-261, 2015.

21. Guan S, Tang Q, Liu W, Zhu R and Li B: Nobiletin Inhibits PDGF-BB-induced vascular smooth muscle cell proliferation and migration and attenuates neointimal hyperplasia in a rat carotid artery injury model. Drug Dev Res 75: 489-496, 2014.

22. Tolva V, Mazzola S, Zerbi P, Casana R, Albertini M, Calvillo L, Selmin F and Cilurzo F: A successful experimental model for intimal hyperplasia prevention using a resveratrol-delivering balloon. J Vasc Surgp ii: S0741-S5214, 2014.

23. Liu Y, He J, Chen X, Li J, Shen M, Yu W, Yang Y and Xiao Z: The proapoptotic effect of formononetin in human osteosarcoma cells: Involvement of inactivation of ERK and Akt pathways. Cell Physiol Biochem 34: 637-645, 2014.

24. Huang X, Jin Y, Zhou D, Xu G, Huang J and Shen L: IQGAP1 promotes the phenotypic switch of vascular smooth muscle by myocardin pathway: A potential target for varicose vein. Int J Clin Exp Pathol 7: 6475-6485, 2014.

25. Song Y, Long L, Zhang N and Liu Y: Inhibitory effects of hydroxysafflor yellow A on PDGF-BB-induced proliferation and migration of vascular smooth muscle cells via mediating Akt signaling. Mol Med Rep 10: 1555-1560, 2014.

26. Song MC, Park J and Kim TJ: Diethylstilbestrol induces arrest of rat vascular smooth muscle cell cycle progression through downregulation of cyclin D1 and cyclin E. Mol Cell Biochem 360: 103-109, 2012. 
27. Ding Q, Chai H, Mahmood N, Tsao J, Mochly-Rosen D and Zhou W: Matrix metalloproteinases modulated by protein kinase $\mathrm{C} \varepsilon$ mediate resistin-induced migration of human coronary artery smooth muscle cells. J Vasc Surg 53: 1044-1051, 2011.

28. Guo L, Ning W, Tan Z, Gong Z and Li X: Mechanism of matrix metalloproteinase axis-induced neointimal growth. J Mol Cell Cardiol 66: 116-125, 2014.

29. Toker A: Achieving specificity in Akt signaling in cancer. Adv Biol Regul 52: 78-87, 2012.

30. Vasudevan KM and Garraway LA: AKT signaling in physiology and disease. Curr Top Microbiol Immunol 347: 105-133, 2010.

31. Liu D, Liu J, Lin B, Liu S, Hou R, Hao Y, Liu Q, Zhang S and Iwamori $\mathrm{M}$ : Lewis y regulate cell cycle related factors in ovarian carcinoma cell RMG-I in vitro via ERK and Akt signaling pathways. Int J Mol Sci 13: 828-839, 2012.
32. Park ES, Kang SI, Yoo KD, Lee MY, Yoo HS, Hong JT, Shin HS, Kim B and Yun YP: Camptothecin inhibits platelet-derived growth factor-BB-induced proliferation of rat aortic vascular smooth muscle cells through inhibition of PI3K/Akt signaling pathway. Exp Cell Res 319: 982-991, 2013.

33. Iida M, Tanabe K, Kozawa $\mathrm{O}$ and Iida $\mathrm{H}$ : Differential effects of intravenous anesthetics on PDGF-BB-induced vascular smooth muscle cell migration. Cell Physiol Biochem 33: 1827-1837, 2014.

34. Son JE, Jeong H, Kim H, Kim YA, Lee E, Lee HJ and Lee KW: Pelargonidin attenuates PDGF-BB-induced aortic smooth muscle cell proliferation and migration by direct inhibition of focal adhesion kinase. Biochem Pharmacol 89: 236-245, 2014. 